\title{
Application of functional neuromuscular stimulation to children with spinal cord injuries: candidate selection for upper and lower extremity research
}

\author{
R J Triolo PhD, ${ }^{1}$ R R Betz MD,${ }^{2,3}$ M J Mulcahey MS OTR/L, ${ }^{2}$ E R Gardner MS PT ${ }^{2}$ \\ ${ }^{1}$ Department of Orthopaedic Surgery and Biomedical Engineering, Case Western Reserve \\ University, Cleveland, Ohio, USA; ${ }^{2}$ Philadelphia Unit of Shriners Hospitals for Crippled \\ Children, Philadelphia, PA 19152, USA; ${ }^{3}$ Department of Orthopaedic Surgery, Temple \\ University Hospital, Philadelphia, PA, USA.
}

This paper summarizes the results of screening for participation in research programs involving functional neuromuscular stimulation (FNS). It examines the characteristics of a group of children and teenagers with spinal cord injuries (SCI) identified as potential candidates for FNS as defined by the rigorous inclusion criteria of the research studies. One hundred and thirteen children and teenagers under the age of 20 with cervical, thoracic or lumbar level spinal cord injuries were examined for inclusion in an experimental program of FNS to provide standing, walking, or prehension. Although biased towards adolescents with complete midthoracic and midcervical injuries, the age, sex, injury level, etiology, and neurological status of the sample coincided with previously published reports and consisted predominantly of teenage males injured in motor vehicle or sports related accidents. Approximately half of the individuals examined were physically appropriate for research participation without preparatory intervention. Treatment options to prepare individuals for FNS were identified in $25 \%$ of those considered inappropriate at the initial evaluation, indicating that the potential user population of clinical systems may be larger than estimates obtained from research applications. Peripheral denervation was the single most prevalent physical impediment to the application of FNS. Although the incidence of lower motor neuron (LMN) involvement was similar in subjects with tetraplegia and paraplegia, those with cervical lesions more frequently exhibited other medical complications that interfered with the application of FNS. Surgical procedures involving transfer of paralyzed but excitable muscles were identified in almost one third of the candidates with tetraplegia who were excluded due to denervation. Of those physically appropriate, psychological factors eliminated several candidates from consideration. Such concerns may also be addressed with suitable intervention in preparation for the clinical application of FNS. Almost $50 \%$ of those appropriate for FNS research elected to participate in the programs, with those declining citing the hospitalization, time and travel commitments as the primary factors influencing their decisions. Results suggest that FNS for standing, walking and hand grasp may be an option for a significant percentage of the pediatric SCI population.

Keywords: spinal cord injury; FNS; neuroprostheses; pediatrics; rehabilitation.

\section{Introduction}

If the neurological damage resulting from a traumatic spinal cord injury is confined to the upper motor neuron, then a small electrical current applied to the peripheral nerve will generate a contraction of its associated muscle. Functional neuromuscular stimulation refers to the activation and coordination of several muscles in this manner to produce a useful, purposeful movement, rather than to achieve a purely 
therapeutic goal such as increasing or maintaining joint range of motion or restrengthening weak or atrophied muscles. The technology has the potential to provide or enhance standing, walking or upper extremity abilities, thereby increasing the number of options available to its users in their pursuit of independence in vocational and avocational activities.

Young people may be at a greater risk of spinal cord injury than any other segment of the population, since injuries occur most frequently in persons between the ages of 15 and 20 years. ${ }^{1-10}$ As trauma care becomes more sophisticated and medical management improves, young people with SCI also demonstrate the best survival rates and life expectancies. Patients between the ages of 10 and 19 have survival rates approaching those of the general population and higher than all other individuals with traumatic injuries. $^{7}$ The life expectancy of a person sustaining an injury at 20 years of age has also been shown to be significantly higher than that of an individual older than 40 at the time of injury. ${ }^{4,9}$ Therefore, FNS may have a greater impact on the lives of people with SCI under the age of 20 than any other segment of the population. As FNS approaches widespread clinical use, it becomes increasingly important to understand the characteristics of children, teenagers and young adults wtih SCI in order to design systems that are appropriate for those individuals who may eventually become long term users of the technology.

For these reasons, researchers at the Philadelphia Unit of Shriners Hospitals, together with Case Western Reserve University, have been investigating the application of FNS to children, adolescents and young adults with SCI. This paper summarizes the results of the screening process used to identify candidates for research programs designed to develop and test FNS devices for standing, walking or prehension in young people under 18 years of age. It represents an initial attempt to identify the segment of the pediatric SCI population which may contain the most likely candidates for research-grade FNS systems and to understand the factors that may interfere with their administration.

\section{Literature review}

\section{Functional neuromuscular stimulation in $S C I$}

FNS systems have ranged from simple, switch activated single channel devices intended to correct foot drop to complex microprocessor controlled devices for standing, walking, or grasp and release. ${ }^{11-12}$ Stimulation has been delivered via surface electrodes, intramuscular wire electrodes that exit the body percutaneously, or implantable systems that utilize epimyseal or nerve cuff electrodes and communicate with an external controller through a radio frequency link. Still other systems have combined FNS with varying degrees of external bracing to constrain the motions generated by the stimulated muscles, simplify control, reduce the number of stimulation channels, or provide mechanical support. ${ }^{13,14}$ Most participants in standing and walking protocols have had complete thoracic lesions between levels T6 and T12. Individuals with incomplete thoracic lesions have also received attention by various researchers. ${ }^{11,12,15}$

For standing, electrodes have most often been applied to the surface of the anterior thigh to excite all heads of the quadriceps simultaneously. ${ }^{11.16 .17}$ Subjects are trained to maintain balance by adopting a 'modified C' posture with the lumbar spine in lordosis and the hips fully extended. Range of motion limitations such as hip flexion contractures, or other orthopaedic complications such as excessive spine curvatures, restrict the usefulness of this posture. Stepping has been demonstrated in individuals with complete paraplegia by applying additional channels of stimulation to one of several sites on the lower extremity which elicit a flexion withdraw reflex. The reflex tends to vary with time and activation history, however, and is often weak or nonexistent. More complex systems for lower extremity function have utilized percutaneous intramuscular electrodes to activate individual muscles, rather than rely on reflex patterns. ${ }^{18} \mathrm{Up}$ to 48 channels of stimulation have been applied ot the muscles controlling the trunk, hip, thigh, knee and ankle to generate standing, walking and 
stair climbing motions. Currently, such lower extremity applications still rely on the use of assistive devices and upper extremity exerti ' $n$ for balance and support, and tend to be energy inefficient. ${ }^{11-12}$

Although the applicability of FNS to individuals with cerebral palsy, ${ }^{19}$ high (C4) ${ }^{20-22}$ or low (C6 and C7) cervical injuries $^{23}$ are currently under investigation, upper extremity applications have concentrated primarily on providing prehension and release to persons with midcervical level tetraplegia. ${ }^{23-29}$ The most advanced and well reported system employs 16 channels of percutaneous intramuscular stimulation to activate the finger flexors, extensors, and thumb muscles. The system is capable of generating two types of prehension patterns: palmar grasp and lateral pinch. Selection and control of the grasps are provided by a single command source, which is usually the position of the contralateral shoulder. Surgical procedures have been prescribed to augment the function of the system, such as stabilization of the interphalangeal joint of the thumb or synchronization of the fingers with tenodeses of the flexor or extensor tendons. ${ }^{26-29}$

With notable exceptions, ${ }^{20-23}$ efforts to provide prehension and release have focused primarily on people with $\mathrm{C} 5$ and weak C6 level injuries because they are unable to benefit from conventional surgical interventions, such as voluntary tendon transfer surgery or orthotic devices. They also retain sufficient proximal control to position their hands in space for functional activities, which increases the likelihood that FNS-produced grasps may be useful. ${ }^{24-26}$

\section{Peripheral denervation}

While it is possible to stimulate muscle tissue directly, most FNS applications rely on activating the peripheral nerve, which is much more excitable. An intact lower motor neuron (LMN) is essential if low levels of current are to be used to generate a strong and repeatable muscular contraction. One study of 24 individuals with injuries between C5 and L3 reported denervation in over $45 \%$ of those patients with thoracolumbar
(T12-L3) lesions and over 7\% of those patients with thoracic (T1-11) lesions, although no evidence of lower extremity peripheral denervation was observed in any of the patients with cervical level injuries. ${ }^{30,31}$ The absolute number of individuals in each group was not reported. In another study of 24 patients with injuries between levels $\mathrm{C} 4$ and $\mathrm{C} 7$, peripheral innervation to the muscles required for prehension with FNS was found to be intact in a majority of instances. ${ }^{32}$ Participants in that investigation ranged from 14 to 30 years and all had been examined within 1 year post injury. The status of the lower motor nerve was assessed via observation of reflex activity, response to surface stimulation, strength-duration testing, or EMG analysis. Over $90 \%$ of the finger flexors and almost $80 \%$ of the finger extensors were found to be totally or partially innervated. Denervation of the finger flexors and extensors was most common with $\mathrm{C} 5$ level injuries, and one out of three individuals with $\mathrm{C} 4$ level injuries exhibited denervation of the biceps.

\section{Candidacy for lower extremity FNS applications}

The patient selection criteria for a clinical program of standing and walking with surface FNS at the University Rehabilitation Institute in Ljubljana, Slovenia included four factors: injury level, neurological status, physiological or metabolic impairments, and physical limitations. ${ }^{15}$ Functional ability and injury level were considered when evaluating individuals with complete lesions. FNS alone was prescribed for people with lesions between T4 and T12. Trunk instability and the potential of developing spine deformities in volunteers with injuries above $\mathrm{T} 4$ indicated a combination of FNS and passive bracing. Application of FNS to individuals with injuries below T12 was often precluded by LMN involvement and bracing alone was prescribed for such individuals. Patients with incomplete lesions were evaluated based entirely on function, without reference to injury level. Neurological status included an assessment of LMN involvement. Excessive spasticity or peripheral denervation of the knee exten- 
sors contraindicated the use of stimulation. Patients were eliminated from consideration for FNS walking if a withdraw reflex could not be elicited repeatedly. Abnormalities in the heart rate, blood pressure, EKG, or the inability to tolerate exhaustion were contraindications to FNS, and range of motion, skin integrity, and joint stability were also part of the physical examination.

Applying these criteria to 500 patients with SCI resulted in the identification of 54 individuals with complete injuries and 26 people with incomplete injuries as candidates for FNS, representing $16 \%$ of the SCI population admitted to the Institute. ${ }^{15,16}$ Although the sample was not typical or statistically unbiased, it was offered as a preliminary estimate of the user population. Fifty patients with complete injuries, or $10 \%$ of the original sample, completed the standing and walking program. Participants were distributed according to sex in a similar manner to that reported for individuals with SCI in the United States $(80 \%$ male, $20 \%$ female)..$^{1-9}$ Thirty percent of the participants dropped out after standing or walking for 3 or more months at home, citing the inconvenience of donning and doffing the four channel surface stimulation system as reason for withdrawal. All patients with lesions between $\mathrm{T} 4$ and $\mathrm{T} 12$ were able to stand with FNS, but only half were able to walk because of undetected neural involvement or partial denervation. Three quarters of patients with T4 and T5 lesions, and half of those with lesions from T6 to T11, were able to produce walking movements.

A study conducted at the Rehabilitation Institute of Chicago (RIC) and the Illinois Institute of Technology (IIT) used similar evaluation criteria and methods to produce standing in individuals with SCI. ${ }^{33.34}$ User population estimates were obtained from reviewing the charts of 192 patients. Inclusion in a program of FNS standing with a two-channel surface system was restricted to individuals who met the profile summarized in Table I. The average age of the sample was 33 years, and males again outnumbered females 4 to 1 . According to these criteria, 20 out of the 192 candidates were considered to be appropriate for FNS, implying that approximately $10 \%$ of all individuals
Table I RIC/IIT evaluation criteria for lower extremity FNS

1 Injury level between $\mathrm{T} 4$ and $\mathrm{T} 12$

2 No upper extremity involvement

3 Prescription of knee-ankle-foot orthoses

4 No associated medical problems

5 No history of substance abuse

6 Age between 18 and 50 years

with paraplegia would be potential users of a stimulation system for standing.

This may be an underestimate of the user population because of the stringent nature of several of the inclusion criteria. Addressing associated medical or psychological problems with appropriate treatment or therapy may have enlarged the estimate. In particular, relaxing the age restriction, or eliminating the requirement of a kneeankle-foot orthosis (KAFO) prescription, may have resulted in an increased number of FNS users. ${ }^{34}$ No allowances were made for the possibility of preparatory interventions that might precede application of FNS. The estimate of the user population was also based solely on a chart review and candidates were not examined personally by the investigators. The RIC/IIT and Ljubljana studies may have been limited by the acceptability of surface stimulation systems.

\section{Candidacy for upper extremity FNS applications}

Although current efforts are directed exclusively toward implantable technology, the inclusion criteria published for the neuroprosthetic system developed at Case Western Reserve University (CWRU) represented a staged approach to the application of FNS. Candidates were expected to progress from a device using percutaneous electrodes to a combination of stimulation and hand surgery to enhance performance, and finally to a fully implanted system. ${ }^{26}$ Patient selection was based on physiological and psychosocial criteria, as well as an assessment of independence following rehabilitation. To date, over 27 individuals with C5/C6 lesions have been provided with prehension and release via percutaneous 
stimulation, 10 have had augmentative surgery and seven have progressed to the implanted system. ${ }^{26-29}$ More recently, the percutaneous stages have been eliminated and augmentative surgery has been performed during the same procedure in which the implanted stimulator is installed. The number of potential candidates examined to identify system users, and relative frequency of physical, psychosocial, or personal barriers to participation has not been reported.

The physiological and psychosocial criteria for acceptance into the percutaneous CWRU program ${ }^{25,26}$ are given in Table II. The criteria applied to a candidate's support mechanisms also included the ability and willingness of the patient and attendant to maintain skin interfaces, don and doff the system, set up evening exercise programs, provide transportation to and from the hospital, and use the system regularly in accordance with the research protocols.

In the event that the primary muscles required for stimulation of palmar and lateral grasps are denervated, it may be possible to intervene surgically. The transfer of the tendon of an innervated but paralyzed muscle to that of a denervated prime mover has been suggested as a method to obviate the problems encountered with LMN involvement which would otherwise eliminate a patient from consideration for FNS. ${ }^{26-29}$ No attempt has been made to estimate the number of individuals with peripheral denervation who would become FNS candidates after such surgery.

\section{Psychological and social criteria}

Although no assessment tools have been validated expressly for the psychological screening of FNS candidates, coping skills, the ability to tolerate uncertainty, and the ability to provide informed consent have been identified as key elements in the emotional makeup of successful FNS research participants. ${ }^{35}$ The Minnesota Multiphasic Personality Inventory, Shipley Institute of Living Scale, the Wide Range of Achievement Test Reading Level II, the Beck Depression Inventory and Acceptance of Disability Scale have been used to assess mental status and problems with relationships, intelligence and cognition, verbal comprehension, depression and emotional disruption, and values associated with disability adjustment in FNS candidates. A retrospective analysis of several participants in the surface standing program at the RIC showed that psychological testing could

Table II Inclusion criteria for CWRU percutaneous neuroprosthetic hand system

\begin{tabular}{|c|c|}
\hline Physiologic criteria & Psychosocial criteria \\
\hline 1 Stable neurological status & $\begin{array}{l}1 \text { Adequate ability to cope with the } \\
\text { disability }\end{array}$ \\
\hline $\begin{array}{l}2 \text { Absence of medical problems (infections, diabetes, } \\
\text { chronic skin breakdown, heart disease, uncontrolled } \\
\text { spasticity) }\end{array}$ & $\begin{array}{l}2 \text { Motivated and able to identify } \\
\text { realistic functional goals }\end{array}$ \\
\hline 3 Intact vision & 3 Normal cognitive function \\
\hline $\begin{array}{l}4 \text { Complete physiological rehabilitation (good sitting } \\
\text { balance and voluntary strength, minimal upper extremity } \\
\text { contractures) }\end{array}$ & $\begin{array}{l}4 \text { Willingness to become an active } \\
\text { research participant }\end{array}$ \\
\hline 5 Minimal hypermobility or joint laxity & \\
\hline 6 No hypersensitivity in the instrumented hand & \\
\hline $\begin{array}{l}7 \text { Adequate proximal control to position the hand for } \\
\text { function }\end{array}$ & \\
\hline $\begin{array}{l}8 \text { Adequate wrist strength (grade } 4 \text { ) or static support to } \\
\text { stabilize the hand }\end{array}$ & \\
\hline 9 Intact innervation of key muscles & \\
\hline 10 Not a candidate for voluntary tendon transfers & \\
\hline
\end{tabular}


identify individuals who had failed to complete the research program. The psychological testing was repeated prospectively on candidates prior to admission to the program and confirmed the projection of future success with FNS standing. ${ }^{17}$

The danger of using psychological testing to predict success with FNS lies in obtaining a false negative result, that is, excluding a candidate from consideration who may in fact be successful. It may be possible to offer FNS systems to people while concurrently addressing their psychosocial needs with the necessary interventions. An additional danger arises from applying psychological criteria in the selection of FNS participants in the absence of reliability studies of such assessments in the SCI population. The values, roles, emotional make-up and process of adjusting to an injury require attention when planning or providing any type of intervention, especially when it involves experimental modalities in a pediatric population. Basing the administration of FNS systems on such incompletely understood phenomenon may artificially and inappropriately restrict the availability of the technology.

\section{Methods}

Data for this study were collected from a convenience sample of 113 children (birth to 12 years) and teenagers (13 to 19 years) with spinal cord injuries. Approximately $55 \%$ of the sample were 1 year or more post injury at the time of the screening exam. All were admitted to the Philadelphia Unit of Shriners Hospitals for evaluation by the authors. The primary purpose of the examination was to identify children willing and appropriate to participate in experimental programs of FNS to provide standing and walking or prehension and release. In addition to the patient population of the Philadelphia Unit, candidates for both upper and lower extremity research programs were also identified by the SCI Units of Shriners Hospitals in Chicago and San Francisco, solicited by the rehabilitation therapies of other Shrine Hospitals, or referred by unaffiliated health care professionals and rehabilitation institutions. Age-appropriate referrals were also made by the FES Information Center at Case Western Reserve University. On occasion, candidates contacted the authors directly after becoming aware of the research program independently. The Philadelphia Unit of Shriners Hospitals also conducts SCI clinics in Greenville, South Carolina and San Juan, Puerto Rico. Candidates for both research programs identified at these clinics were also examined. Age at injury, etiology, neurological status, race, sex, and other demographic data were collected and collated for each referral.

The criteria for the lower extremity FNS program at Shriners Hospitals for Crippled Children (SHCC) are presented in Table III. Children with paraplegia who had been treated at the Philadelphia Unit prior to 1986 were identified from the medical records and readmitted for examination. From 1986 to 1991, individuals with spastic paralysis treated at Shriners who were likely standing and walking candidates underwent the complete screening procedure. A week long screening visit including appointments with physical and occupational therapy (PT, OT), nursing (N), recreational therapy (RT), social services (SS), and the medical staff was scheduled for each candidate for lower extremity FNS to assess thoroughly their physical and psychosocial condition.

Table III SHCC inclusion criteria for lower extremity FNS

1 Age up to 20 years

2 Motor complete spinal cord injury below T1

3 No LMN involvement of the quadriceps, gluteal or hamstring muscles

4 Functional independence in ADLs, wheelchair management and transfers

5 Range of motion within functional limits for standing and walking with KAFOs (hip and knee flexion contractures less than $20^{\circ}$ )

6 Ability to stand and ambulate in braces

7 No outstanding orthopaedic involvement (scoliosis, history of spontaneous fracture, etc)

8 No outstanding medical problems (uncontrolled spasticity, skin involvement, etc)

9 No outstanding psychosocial involvement that would negatively influence research participation 
During this time, the operation and maintenance of the FNS systems, as well as the percutaneous electrode implantation procedure, were explained using demonstrations, videotape presentations, and written materials.

Lower extremity screening tests included evaluations of functional status and equipment (PT, OT), assessments of cognitive and perceptual ability (OT), administration of the Leisure Diagnostic Battery (RT), and determination of general health including skin integrity, bowel and bladder management $(\mathrm{N})$, along with a complete medical examination including plane $\mathrm{x}$-rays to document orthopaedic status and bone age. A formal psychosocial evaluation of the child and family (SS) was also performed. The innervation status of individuals with paraplegia was determined by performing a strength-duration test on the quadriceps, gluteal and hamstring muscles bilaterally, unless a critical muscle was clearly and unambiguously denervated. All tests were completed independently by different members of the clinical or research teams. At the conclusion of the screening process, a summation meeting was held and a collaborative decision was made regarding whether or not the child was a candidate for FNS.

Every child with a cervical level spinal cord injury treated at Shriners Hospital in Philadelphia from 1989 to 1991 was examined and evaluated for research participation. A three day screening admission was arranged for each upper extremity candidate, and consultations were obtained from social services, medical staff, and an independent hand surgeon. Inclusion criteria for the upper extremity FNS program were adapted from those reported by CWRU and are summarized in Table IV. Modifications included the addition of age-appropriate evaluations and an upper age limit.

Upper extremity innervation was determined by first testing the response to surface stimulation. Full strength-duration curves were obtained only for those muscles with questionable, nonspecific, or weak responses. If the peripheral nerve was not found to be intact, the individual was further evaluated to determine whether a
Table IV SHCC inclusion criteria for upper extremity FNS

1 Age up to 20 years

2 SCI resulting in C5-6 tetraplegia

3 Optimal independent function in ADLs

4 Functional sitting balance (or mechanisms to substitute for lack of trunk control) and good sitting tolerance

5 Independent wheelchair mobility

6 Independent trial of traditional orthotic devices

7 Stable neurological status

8 No outstanding medical problems (skin involvement, uncontrolled disreflexia, etc)

9 Intact vision, audition, cognition and perception

10 Appropriate upper extremity status (adequate proximal control and balance for bilateral function, full passive range of movement (ROM), no intolerable hypersensitivity, intact LMN status)

11 Not a candidate for active tendon transfer surgery

12 No outstanding psychosocial involvement that would negatively influence research participation

paralyzed but stimulatable muscle was available that could be transferred to accomplish the same function as the denervated muscle.

Candidates for both upper and lower extremity applications were classified according to the algorithm depicted in Figure 1. Initial consideration was given to the physical status of each individual. Physically appropriate candidates were evaluated and further classified according to psychosocial status. A comprehensive assessment focusing on family structure and demographics, attitudes and feelings toward the child, perceptions of the child's strengths and difficulties, coping mechanisms, expectations of the FNS device and research participation, support systems, and family dynamics was administered. In addition, the family APGAR Questionnaire ${ }^{36}$ was administered to measure satisfaction with family assistance, mutuality of communication and problem-solving, freedom to change roles and grow emotionally, intimacy and emotional interaction, and time commitments made by family members. The social worker assigned to the patient made the final 


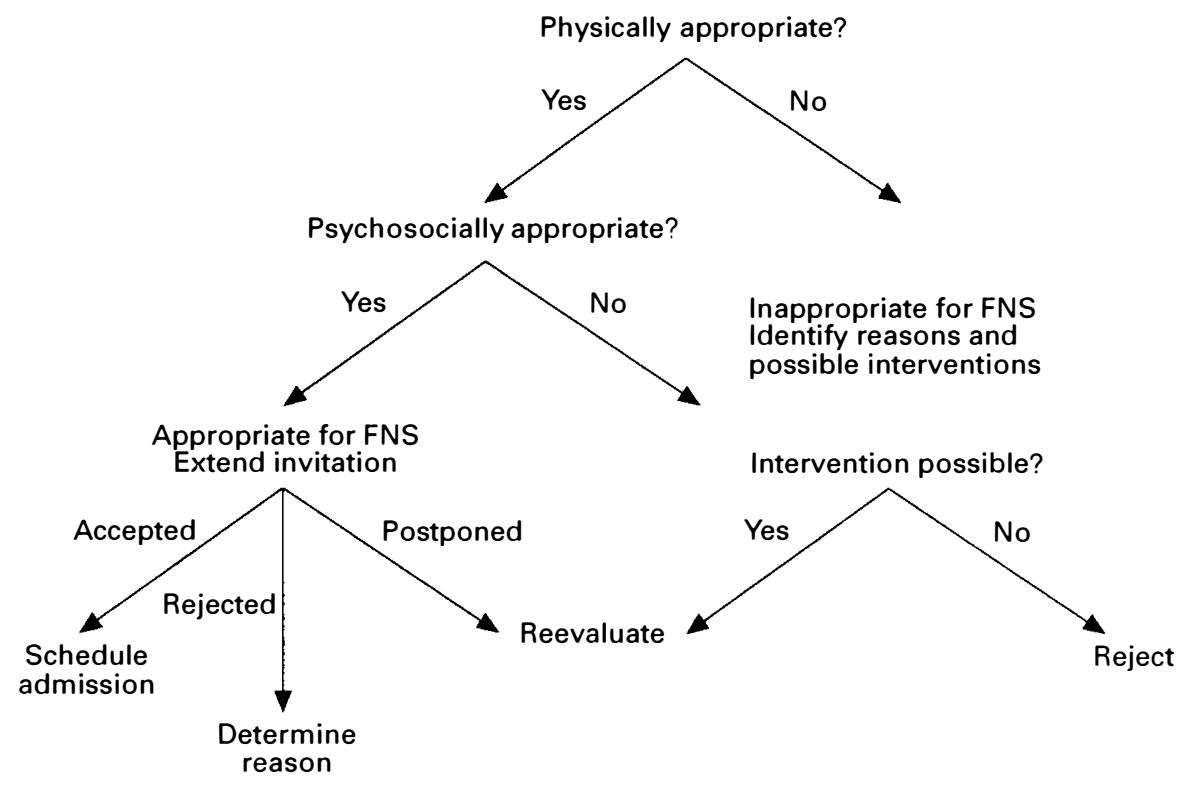

Figure 1 Classification algorithm for FNS candidates.

determination of psychosocial status and made recommendations regarding research participation.

The relative percentages of all candidates who were physically and psychosocially appropriate, physically appropriate but with interfering psychosocial issues, or physically inappropriate, were tabulated. Interventions which might address the physical or psychosocial impediments to participation were identified for those individuals who did not meet the inclusion criteria. These candidates were scheduled for reevaluation. Individuals who were physically and psychosocially suited for FNS were extended invitations to participate in the research program. As illustrated in Figure 1, the candidate exercised the option to accept, postpone, or decline research participation. Reasons for declining the invitation were recorded.

Results of the screening process for research participation were pooled to offer insight into the nature of the group of research applicants as a whole. Pooled data were expressed as percentages of all individuals examined. Where possible, résults from those with cervical (C4-8) and thoracic/lumbar (T1-L3) injuries were exam- ined separately to allow differential comparisons. When analyzed separately, results were expressed as the percentage of people in each subgroup.

\section{Results}

\section{Subject demographics}

Approximately two thirds of the entire sample were male, a smaller percentage than reported for the general SCI population, ${ }^{7-9}$ but coinciding with preliminary reports in pediatrics. ${ }^{10}$ An interaction between sex and injury level was also observed. While children with paraplegia appeared to be distributed relatively evenly between the sexes, those with cervical level injuries appeared to be predominantly male. Approximately $64 \%$ of all males examined presented with cervical level injuries, as compared to only one third of all females.

Midcervical (C5 and C6) and midthoracic (T5-8) level injuries were the most commonly observed lesions in the sample, as illustrated in Figure 2. The frequency of $\mathrm{C5}$ and C6 injuries is comparable to that expected from the SCI population in general. ${ }^{7-9}$ The frequency of injuries in the 


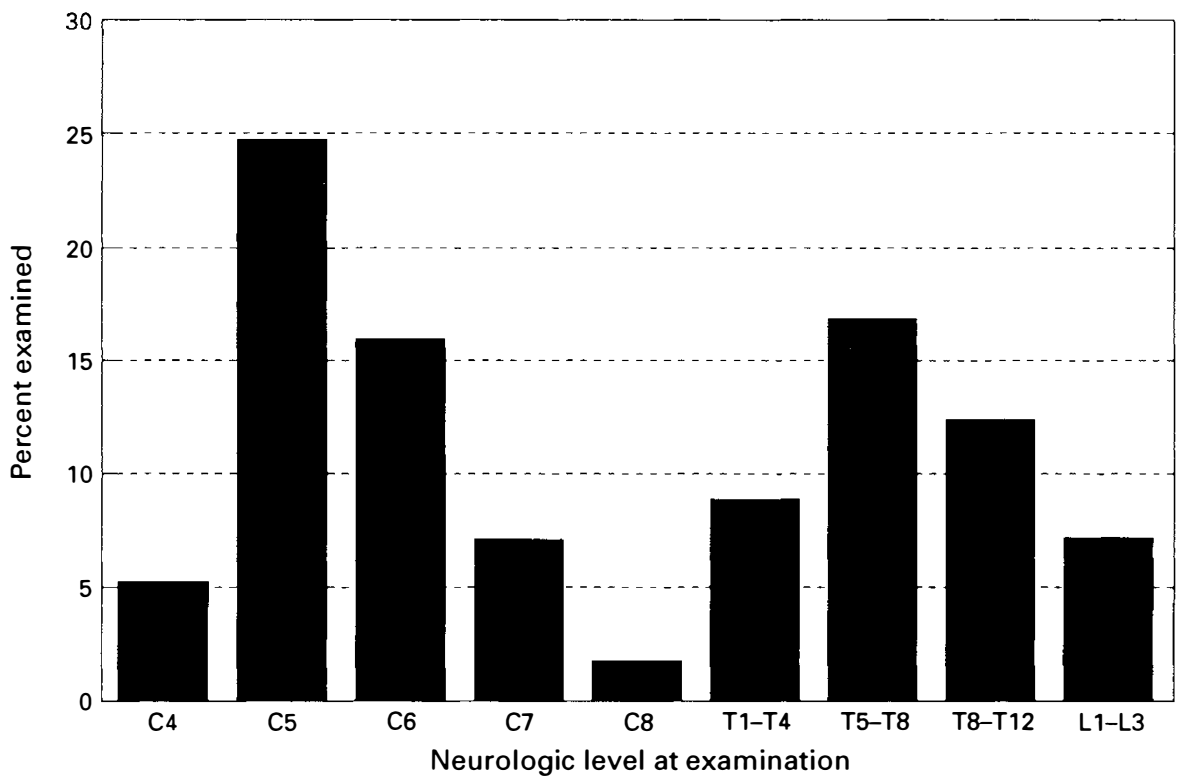

Figure 2 Distribution of injury levels at time of examination $(n=113)$.

midthoracic levels varies slightly from other reports by underrepresenting the prevalence of thoracolumbar level injuries, however. This may be due to the preferential targeting of individuals with midthoracic injuries by referring agencies with knowledge of the inclusion criteria for the research programs.

Almost three quarters of the sample had sustained their injuries while between the ages of 11 and 20. As shown in Figure 3a, the most commonly observed age at injury was between 16 and 20 years, representing almost $40 \%$ of all those examined. This was followed by injuries sustained by teenagers between 11 and 15 years of age, representing over one third of the sample. These results appear to reflect what would be expected from the proportions reported for both pediatric SCI and the SCI population at large. ${ }^{7-10} \mathrm{~A}$ differential presentation of age with respect to injury level is given in Figure 3b. Candidates with cervical level injuries tended to be older, while those with thoracic and lumbar level injuries tended to be more evenly distributed across age. This is in contrast to the pediatric literature reporting that cervical injuries are more common in younger children (birth through 8 years) than older children (9 through 16
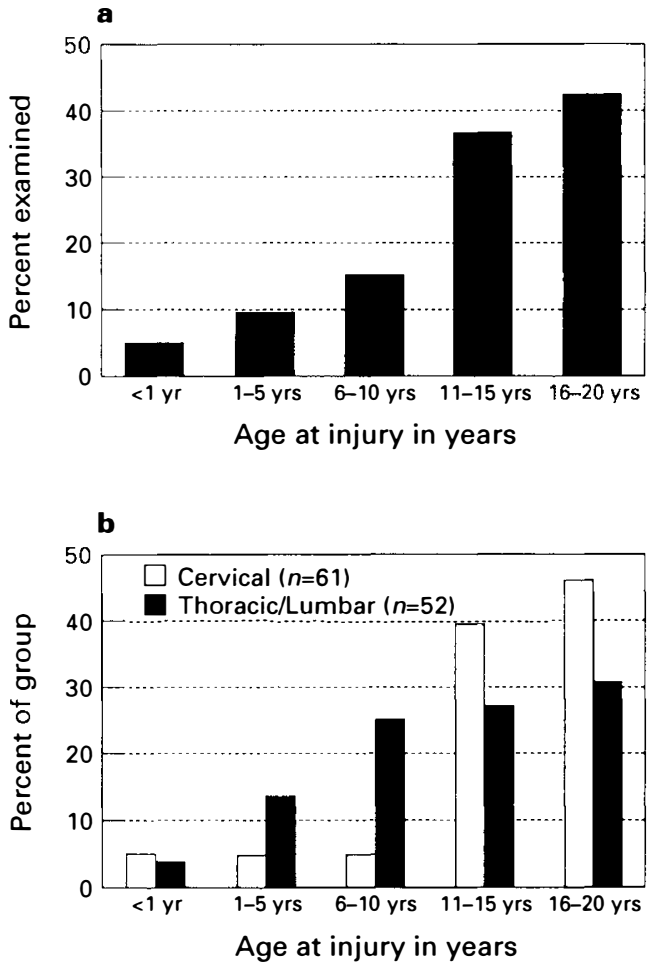

Figure 3 Age at injury for sample pediatric SCI population: (a) all injuries $(n=113)$, (b) as a function of injury level. 
years). ${ }^{10}$ This again may reflect the bias of the authors and referring agencies toward older applicants.

Motor vehicle accidents (MVA) were the most common cause of SCI in the young people examined, as shown in Figure 4a. Over $50 \%$ of all the injuries observed were due to accidents involving an automobile, bus or motorcycle. Diving and sports related injuries were the next most frequently observed causes of SCI. If combined, the MVA and diving/sports groups account for over $75 \%$ of the injuries seen in this sample, following closely the proportions reported in the National SCI Database for the same age group. ${ }^{7}$ Disease, gun shot wounds (GSW), and falls were observed less frequently. The disease category consisted of cases of transverse myelitis (TM) or spinal tumors. Other causes of injury included iatrogenic injury, surgical complication, birth trauma or vascular accident. Although MVAs were the leading cause of both
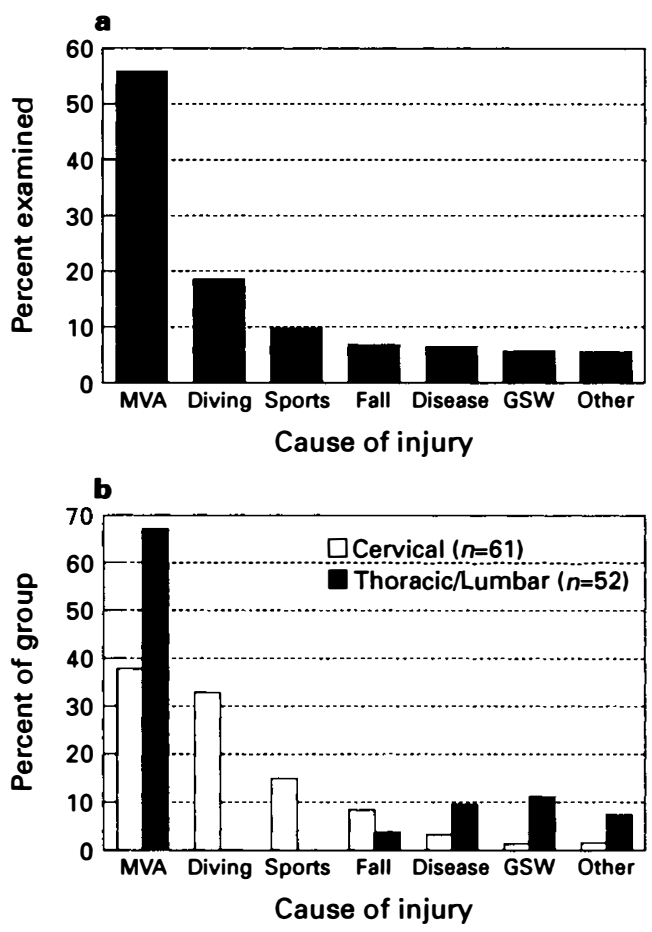

Figure 4 Etiology of SCI in population sample: (a) all injuries $(n=113)$, (b) etiology as a function of injury level. tetraplegia and paraplegia, they resulted in more cases of paraplegia in this sample, as illustrated in Figure 4b. Two thirds of all cases of paraplegia were attributed to a motor vehicle, as compared to $38 \%$ of the cases of tetraplegia. All diving and sports related injuries observed resulted in tetraplegia, while most GSWs yielded thoracic level injuries, which suggests a strong interaction between etiology and injury level. There also appears to be an interaction between etiology and age. No diving, sports, or fall related injuries were observed in children under 10 years of age. Conversely, disease processes such as spinal tumors and TM were observed exclusively in that age group. MVAs were distributed across all ages, but tended to be heavily skewed toward older children and teenagers.

Although the sample was not random and may be biased towards individuals meeting the research selection criteria, the characteristics of the subjects included in this study generally agree with reports describing younger segments of the SCI population.

\section{FNS candidacy}

Over $45 \%$ of all individuals examined were found to be physically appropriate for FNS and free from orthopaedic complications or LMN involvement which would preclude stimulation. The majority of the barriers to the application of FNS appeared to be physical in nature. Of those found to be physically suited for some form of FNS, over $80 \%$ exhibited no psychosocial problems which might limit research participation. These results imply that more than one third of all people included in this study met the physical and psychosocial criteria for FNS at the time of examination.

Figure 5 contains a differential analysis of candidates for upper and lower extremity FNS research programs. As seen in Figure $5 \mathrm{a}$, over $60 \%$ of those individuals with thoracic or lumbar lesions passed the physical examinations and were medically fit for the application of FNS for standing or walking. Few instances of psychosocial involvement severe enough to limit participation were observed and close to $95 \%$ of the 
a

Fail

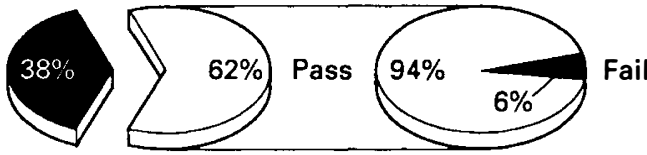

Physical examination ( $n=52)$

b

Fail

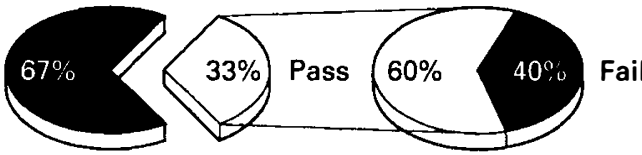

Physical examination ( $n=61$ )

Psychosocial examination $(n=20)$

Figure 5 Comparative results of FNS screening: (a) candidacy for lower extremity FNS applications $(n=52)$, (b) candidacy for upper extremity FNS applications $(n=61)$.

physically appropriate candidates were also well suited psychosocially. This suggests that over $55 \%$ of all individuals examined with thoracic or lumbar level injuries would be able to take advantage of lower extremity FNS as defined in the research program.

A similar analysis for individuals with cervical level injuries is given in Figure 5b. Fully one third of the candidates examined with tetraplegia were physically well suited for FNS. The majority of these individuals also met the psychosocial criteria. Sixty percent of those who were physically appropriate exhibited no significant psychosocial problems severe enough to prevent participation in the FNS research program. The types of psychosocial factors which were observed included depression, anorexia nervosa, drug or substance abuse, lack of attendant or family support or other environmental factors. These results indicate that almost one quarter of the individuals with tetraplegia were both physically and psychosocially appropriate for FNS as defined by the selection criteria at the time of the initial screening.

The principal physical and medical complications which would prohibit the applica- tion of FNS are summarized in Figure 6a. Occasionally these conditions were not isolated but were present in combination. In these cases, the candidate was categorized according to what was considered to be the major limiting factor. The most common physical reason for not recommending FNS appears to be LMN involvement. Almost $40 \%$ of the individuals found to be physically inappropriate for FNS exhibited denervation of the primary muscles for grasp and release or for standing and stepping. Close to $30 \%$ of those unsuited for the FNS research program were candidates for more traditional treatment such as voluntary muscle transfers to augment grasp. Orthopaedic complications which prevented the administration of FNS included the presence of contractures, and hip subluxations. The physical problems listed as 'other' included one instance each of skin problems,
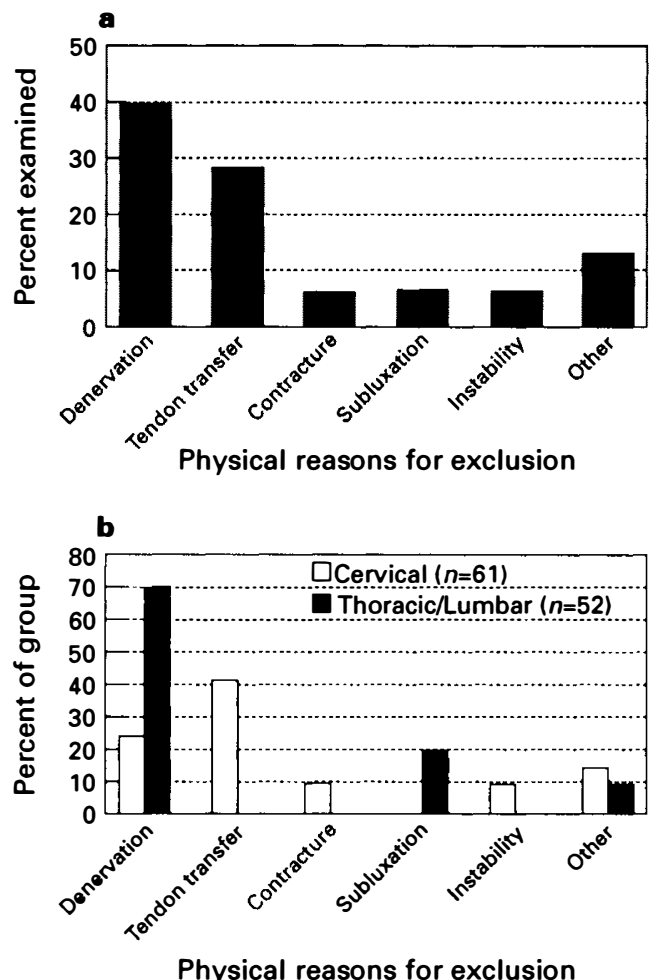

Figure 6 Physical and medical complications interfering with FNS: (a) pooled contraindications to FNS $(n=113)$, (b) contraindications to FNS as a function of injury level. 
hypersensitivity or abnormal sensation, and neurological instability or spontaneous return of function.

A differential comparison of the physical impediments to participation in the FNS programs for upper and lower extremity applications is given in Figure 6b. Of the individuals with paraplegia categorized as physically inappropriate, almost $70 \%$ showed denervation of the antigravity muscles of the legs. Similarly, peripheral denervation was the leading cause of exclusion for children with tetraplegia. Orthopaedic problems for people with paraplegia included two instances of joint subluxation, while four individuals with tetraplegia exhibited interfering upper extremity contractures. Only individuals with tetraplegia were excluded due to neurological instability or the prescripition of tendon transfers. The 'other' category included two people with thoracic injuries exhibiting recurrent skin problems and adverse sensation. The relative frequencies of these complications may be misleading. The absolute number of individuals with tetraplegia who exhibited peripheral denervation was actually larger than the number of people with paraplegia with $\mathrm{LMN}$ involvement. Because candidates with cervical injuries presented with more medical contraindications than children with thoracic or lumbar injuries, the relative percentage of denervation as the primary impediment to FNS appears to be lower. More children with tetraplegia exhibited peripheral denervation, but more were also disqualified for other medical complications.

Some degree of peripheral denervation was observed in approximately $28 \%(17 / 61)$ of people with cervical level lesions at the time of examination. The injury level, etiology, and extent of LMN involvement are summarized in Table $\mathrm{V}$. Intact innervation was observed more frequently in the finger and thumb flexors, while LMN damage was more common in the extensor muscles. When expressed as a percentage of all similar muslces tested, more than $90 \%$ of all finger and thumb flexors retained intact innervation, while almost $80 \%$ of all finger and thumb extensors remained innervated and would be excitable with FNS. These results are comparable to those previously reported for the adult SCI population. ${ }^{32}$

Approximately $27 \%(14 / 52)$ of the individuals with thoracic or lumbar injuries exhibited partial or complete LMN involvement in the primary muscles of the lower extremities. The injury level, etiology and denervation patterns of these cases are summarized in Table VI. Both children with injuries resulting from transverse myelitis exhibited peripheral denervation as a result of the disease process, although a relationship between the pattern of denervation and etiology is not readily evident. There appears to be an interaction with injury level, however. Over half of the individuals with injuries below T12 exhibited some peripheral denervation in the lower extremities because of the proximity to the cauda equina. ${ }^{31,32}$

Figure 7a summarizes the participation status of the children found to be both physically and psychosocially appropriate. Slightly less than half of the children who were both physically and psychosocially fit for FNS chose to participate in one of the stimulation programs. Approximately 25\% of those well suited for stimulation declined the offer. Reasons for declining reflected the inpatient nature of the research program and the current state of the research-grade FNS systems. They included the necessity to be away from home or interrupt schooling during hospitalization for implantation, training, and data collection, as well as dissatisfaction with the cosmesis of the system or the appearance of the stimulated movements. The separation from home and family and the travel necessary for children from geographically distant areas made some parents postpone the application of FNS. Fifteen percent were interested, but preferred to be admitted into the program upon completion of their high school studies, over their summer vacations, or after the FNS systems were more fully developed and out of the research stage. Several children under the age of 10 were considered too young to tolerate the implantation and other procedures. Others had exceeded the admission age limitation for the research programs by the time their screening examinations were completed and 
Table $\mathbf{V}$ Upper extremity denervation patterns at time of examination

\begin{tabular}{|c|c|c|c|c|c|c|c|c|c|c|c|c|c|c|c|c|c|c|c|c|c|c|c|c|c|c|c|c|}
\hline \multirow[t]{3}{*}{ Subject } & \multirow[t]{3}{*}{ Level } & \multirow[t]{3}{*}{ Cause } & \multicolumn{4}{|c|}{$\begin{array}{l}\text { Finger } \\
\text { Flexors }\end{array}$} & \multicolumn{4}{|c|}{$\begin{array}{c}\text { Finger } \\
\text { Extensors }\end{array}$} & \multicolumn{4}{|c|}{$\begin{array}{l}\text { Thumb } \\
\text { Flexors }\end{array}$} & \multicolumn{4}{|c|}{$\begin{array}{l}\text { Thumb } \\
\text { Extensors }\end{array}$} & \multirow{2}{*}{\multicolumn{2}{|c|}{$\begin{array}{c}\begin{array}{c}\text { Thumb } \\
\text { Adductors }\end{array} \\
\text { AdP }\end{array}$}} & \multicolumn{4}{|c|}{$\begin{array}{c}\text { Thumb } \\
\text { Abductors }\end{array}$} & \multicolumn{4}{|c|}{$\begin{array}{c}\text { Wrist } \\
\text { Extensors }\end{array}$} \\
\hline & & & \multicolumn{2}{|c|}{ FDS } & \multicolumn{2}{|c|}{ FDP } & \multicolumn{2}{|c|}{ EDC } & \multicolumn{2}{|c|}{ EIP } & \multicolumn{2}{|c|}{ FPL } & \multicolumn{2}{|c|}{ FPB } & \multicolumn{2}{|c|}{ EPL } & \multicolumn{2}{|c|}{ EPB } & & & \multicolumn{2}{|c|}{ AbPL } & \multicolumn{2}{|c|}{ AbPB } & \multicolumn{2}{|c|}{ ECRL } & \multicolumn{2}{|c|}{ ECRB } \\
\hline & & & $\mathrm{L}$ & $\mathrm{R}$ & $\mathrm{L}$ & $\mathrm{R}$ & $\mathrm{L}$ & $\mathrm{R}$ & $\mathrm{L}$ & $\mathrm{R}$ & $\mathrm{L}$ & $\mathrm{R}$ & $\mathrm{L}$ & $\mathrm{R}$ & $\mathrm{L}$ & $\mathrm{R}$ & $\mathrm{L}$ & $\mathrm{R}$ & $\mathrm{L}$ & $\mathrm{R}$ & $\mathrm{L}$ & $\mathrm{R}$ & $\mathrm{L}$ & $\mathrm{R}$ & $\mathrm{L}$ & $\mathrm{R}$ & $\mathrm{L}$ & $\mathrm{R}$ \\
\hline 1 & $\mathrm{C} 4$ & MVA & & & & & $X$ & $\backslash$ & $X$ & 1 & & & & & $X$ & 1 & $X$ & 1 & & & $X$ & $\backslash$ & & & $X$ & $\backslash$ & $X$ & $\backslash$ \\
\hline 2 & $\mathrm{C} 4$ & MVA & & & & & $X$ & $X$ & $X$ & $X$ & & & & & $X$ & $X$ & $X$ & $X$ & & & $X$ & $X$ & & & $X$ & $X$ & $\mathrm{X}$ & $X$ \\
\hline 3 & $\mathrm{C} 5$ & MVA & $X$ & $X$ & $X$ & $X$ & $X$ & $X$ & $X$ & $X$ & $X$ & $X$ & $X$ & $X$ & $X$ & $X$ & $X$ & $X$ & $X$ & $X$ & $X$ & $X$ & $X$ & $X$ & 1 & 1 & 1 & $\backslash$ \\
\hline 4 & $\mathrm{C} 5$ & Fall & & & & & $X$ & $X$ & & & & & & & $X$ & $X$ & & & & & & $X$ & & & & & & \\
\hline 5 & $\mathrm{C} 5$ & Fall & & & $X$ & & & & & & $X$ & & $X$ & & & & & & & & & & & & & & & \\
\hline 6 & $\mathrm{C} 5$ & TM & $X$ & $X$ & $X$ & $X$ & $X$ & $X$ & $X$ & $X$ & $X$ & $X$ & $X$ & $X$ & $X$ & $X$ & $X$ & $X$ & $X$ & $X$ & $X$ & $X$ & $X$ & $X$ & $X$ & $X$ & $X$ & $x$ \\
\hline 7 & $\mathrm{C} 5$ & Fall & & & & & $X$ & $X$ & & & & & & & $X$ & $x$ & & $X$ & & & & & $X$ & $X$ & & & & \\
\hline 8 & $\mathrm{C} 5$ & MVA & $X$ & $X$ & $X$ & $X$ & $X$ & $X$ & & & $X$ & $X$ & $X$ & $X$ & $X$ & $X$ & $X$ & $X$ & $X$ & $X$ & & & & & & & & \\
\hline 9 & C5 & Dive & & & & & $X$ & $X$ & $X$ & $X$ & & & & & $X$ & $X$ & $X$ & $X$ & & & $X$ & $X$ & $X$ & $X$ & $X$ & $X$ & $X$ & $X$ \\
\hline 10 & C5 & MVA & & & & & $X$ & $X$ & $X$ & $X$ & & & & & $x$ & $X$ & $X$ & $X$ & & & & & & & & & & \\
\hline 11 & $\mathrm{C} 5$ & Dive & & & & & $X$ & $X$ & & & & & & & $X$ & $X$ & $X$ & $X$ & & & & & & & & & & \\
\hline 12 & $\mathrm{C} 5$ & Sport & $X$ & $X$ & $X$ & $X$ & & $X$ & & & $X$ & $X$ & $X$ & & $X$ & & & $X$ & $X$ & & & $X$ & & & & & & \\
\hline 13 & C6 & MVA & & & & & 1 & 1 & $\backslash$ & 1 & & & & & & & & & & & & & & & & & & \\
\hline 14 & C6 & MVA & & & & & $X$ & $X$ & $X$ & $X$ & & & & & $X$ & $X$ & $X$ & $X$ & & & $X$ & $X$ & & & & & & \\
\hline 15 & C6 & Sport & 1 & 1 & $X$ & $X$ & 1 & 1 & $\backslash$ & $\backslash$ & $\backslash$ & & & $X$ & $X$ & 1 & & & $X$ & $X$ & 1 & $\backslash$ & 1 & 1 & & & & \\
\hline 16 & C6 & MVA & $X$ & & $X$ & & & $X$ & & $X$ & $X$ & & $X$ & & & $X$ & & $X$ & $X$ & & & $X$ & & & & & & \\
\hline 17 & $\mathrm{C} 7$ & Dive & & & & & $X$ & $X$ & $X$ & $X$ & & & & & $X$ & $X$ & $X$ & $X$ & & & $X$ & $X$ & $X$ & $X$ & $X$ & $X$ & $X$ & $X$ \\
\hline
\end{tabular}

$\mathrm{X}=$ complete denervation

$\backslash$ = partial denervation

MVA = motor vehicle accident

$\mathrm{TM}=$ transverse myelitis 
Table VI Lower extremity denervation patterns at time of examination

\begin{tabular}{|c|c|c|c|c|c|c|c|c|c|c|c|c|}
\hline \multirow[t]{3}{*}{ Subject } & \multirow[t]{3}{*}{ Level } & \multirow[t]{3}{*}{ Cause } & \multicolumn{4}{|c|}{$\begin{array}{c}\text { Knee } \\
\text { Extensors }\end{array}$} & \multirow{2}{*}{\multicolumn{2}{|c|}{$\begin{array}{c}\begin{array}{c}\text { Knee } \\
\text { Flexors }\end{array} \\
\text { BF }\end{array}$}} & \multicolumn{4}{|c|}{$\begin{array}{c}\text { Hip } \\
\text { Extensors }\end{array}$} \\
\hline & & & \multicolumn{2}{|c|}{$\mathrm{VM}$} & \multicolumn{2}{|c|}{ VL } & & & \multicolumn{2}{|c|}{ GMax } & \multicolumn{2}{|c|}{ GMed } \\
\hline & & & $\mathrm{L}$ & $\mathrm{R}$ & $\mathrm{L}$ & $\mathrm{R}$ & $\mathrm{L}$ & $\mathrm{R}$ & $\mathrm{L}$ & $\mathrm{R}$ & $\mathrm{L}$ & $\mathrm{R}$ \\
\hline 18 & $\mathrm{~T} 1$ & MVA & & & & & 1 & 1 & 1 & 1 & & \\
\hline 19 & $\mathrm{~T} 8$ & MVA & $X$ & $\mathrm{X}$ & $X$ & $X$ & & & $X$ & $\mathrm{X}$ & $X$ & $\mathrm{X}$ \\
\hline 20 & $\mathrm{~T} 8$ & VA & 1 & 1 & 1 & 1 & & & 1 & 1 & & \\
\hline 21 & T9 & MVA & & & $X$ & $X$ & & & $X$ & $X$ & & \\
\hline 22 & T10 & GSW & $X$ & & 1 & & & & & & & \\
\hline 23 & $\mathrm{~T} 10$ & TM & 1 & X & & & & & & $X$ & & \\
\hline 24 & $\mathrm{~T} 12$ & MVA & $X$ & 1 & 1 & $X$ & & & $X$ & X & & \\
\hline 25 & $\mathrm{~T} 12$ & MVA & $X$ & $X$ & $X$ & $X$ & & & & & & \\
\hline 26 & $\mathrm{~T} 12$ & MVA & & $\mathrm{X}$ & & $X$ & $\mathrm{X}$ & & & $X$ & & $\mathrm{X}$ \\
\hline 27 & $\mathrm{~T} 12$ & MVA & X & $\mathrm{X}$ & $X$ & $X$ & & & & & & \\
\hline 28 & L1 & MVA & $X$ & $\mathrm{X}$ & & & & & & & & \\
\hline 29 & $\mathrm{L1}$ & MVA & 1 & 1 & 1 & $X$ & & & $X$ & $X$ & & \\
\hline 30 & L2 & MVA & 1 & 1 & 1 & 1 & & & $X$ & $X$ & & \\
\hline 31 & L2 & Tumor & & 1 & & & & $X$ & & & & X \\
\hline
\end{tabular}

$\mathrm{X}=$ complete denervation

$\backslash$ = partial denervation

GSW $=$ gun shot wound

MVA = motor vehicle accident

$\mathrm{TM}=$ transverse myelitis

$\mathrm{VA}=$ vascular accident

$\mathrm{VM}=$ vastus medialis

$\mathrm{VL}=$ vastus lateralis

$\mathrm{BF}=$ biceps femoris (long head)

GMax $=$ gluteus maximus

GMed $=$ gluteus medius

were no longer eligible for treatment in the Shriners Hospitals system. In other cases, the travel, especially for children living out of the country, prohibited participation in the Shriners research program. These situations were grouped into the 'other' category and represent externally imposed limitations, rather than a response to the offer of FNS itself.

Figure $7 \mathrm{~b}$ is a differential comparison of the participation status of children with tetraplegia and paraplegia. A larger percentage of the children physically and psychosocially appropriate for lower extremity FNS chose to decline or postpone participation. This may be due in part to several programmatic constraints, such as the inpatient nature of lower extremity FNS program which required research subjects to live at the Philadelphia Unit, in addition to the candidate's perception of the relative benefits of research participation.

\section{Discussion}

Due to the possible presence of an orthopaedic complication, peripheral denervation, and social or economic constraints, not all individual with SCI may be well suited for FNS. Because of the expense, complexity, unknown functional benefits, and high level of maintenance, FNS systems have been primarily experimental in nature and restricted to use in clinical research programs. As such, they have usually been applied to highly motivated adults who meet the rigorous inclusion criteria of a specific 

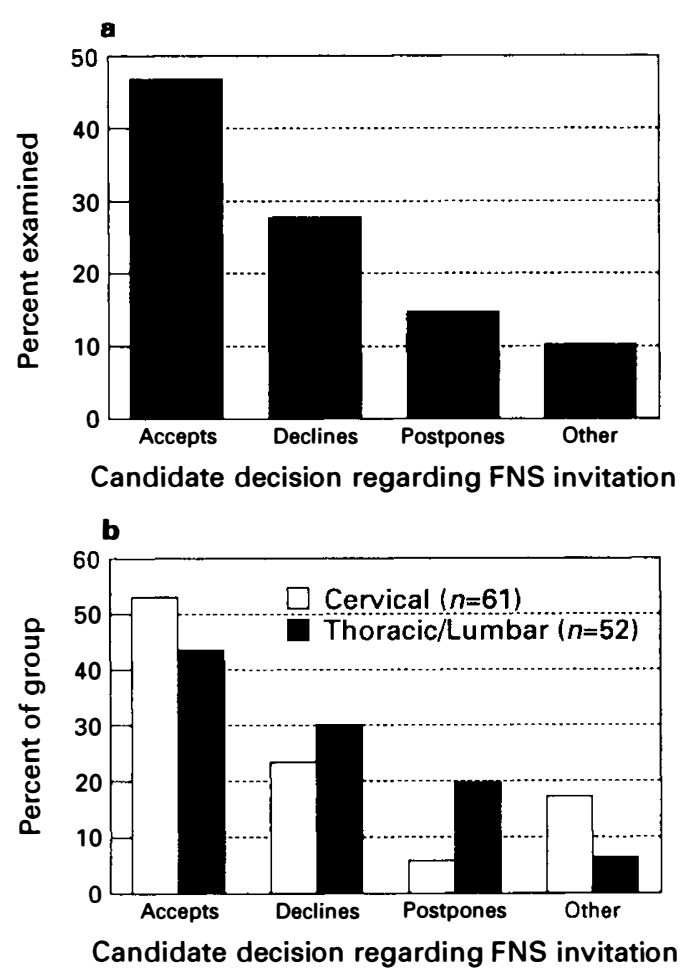

Figure 7 Participation status of appropriate candidates: (a) pooled research participation status $(n=113)$, (b) participation status as a function of injury level.

research study, making the clinical impact of FNS difficult to predict. Not all individuals with SCI who may benefit from FNS may be suited for research applications, and many others may opt not to participate in experimental programs. Until recently, the applicability for the technology to the pediatric SCI population has received little attention.

The results of this study indicate that almost half of the children surveyed could be expected to be both physically and psychosocially suited for FNS in the research setting. They suggest that, even in the absence of any preparatory intervention, a large segment of the pediatric SCI population may be able to take advantage of some form of FNS as systems and protocols for clinical applications become available in the future.

A larger percentage of children with paraplegia met the inclusion criteria for FNS
(58\%) than did children with tetraplegia $(20 \%)$ because of the complicating medical problems and psychosocial issues that typify the postinjury period for individuals with midcervical injuries. This observation highlights an important consideration when interpreting the results of screening for FNS candidacy. The data collected here represent a 'snap shot' of the characteristics of the candidate population. Decisions regarding FNS participation were made based on data collected only at the time of the screening evaluation and without regard to possible medical treatment or therapeutic intervention which might have made an otherwise inappropriate candidate better suited for FNS. Often, simple interventions which would prepare a patient for FNS could be easily identified, such as surgery, splinting, or other therapy to alleviate contractures.

Contractures were the most common orthopaedic problem encountered at the joints. They can best be controlled with medication for spasticity and good daily stretching regimens of the lower extremities in patients with paraplegia and of both upper and lower extremities for patients with tetraplegia. Where ankle equinus is a severe problem, night splinting could be instituted. On occasion, elbow and wrist splinting may also be prescribed. Surgery was recommended for all four individuals who presented with limiting upper extremity contractures. These children may be appropriate for FNS once functional range of motion is achieved. It may be possible to address several of the physical barriers to participation in a similar fashion.

Subluxation and dislocation appear to be common problems at the hip, with $28 \%$ incidence in the pediatric spinal cord injured population. ${ }^{37}$ Control of spasticity in the hip joints is essential along with the prevention of hip flexion and adduction contractures which may contribute to the subluxation. Stretching and early prophylactic bracing may be helpful, along with the use of an abduction splint in the wheelchair. Should subluxation occur, surgical procedures such as soft-tissue releases and bony supplementation are possible. Since hips dislocate posteriorly, the bony coverage would need to be augmented in that region of the 
acetabulum. This procedure was also performed on two participants with hip subluxations who went on to stand with FNS after surgery and rehabilitation.

Spinal curvature occurs in $98 \%$ of patients with spinal cord injury, with $67 \%$ requiring surgery. ${ }^{38}$ In lower extremity applications, spinal curves compromise standing posture and result in excessive use of the arms for support; in upper extremity applications spinal curves interfere with sitting balance and may prohibit the execution of bimanual activities. Early prophylactic bracing should be considered for FNS candidates before the curves begin to interfere with function. Both upper and lower extremity FNS systems can be implemented in the presence of prophylactic bracing, although modifications to either the system or brace may be required. For example, a brace designed to correct sitting posture may need to be customized to allow a patient to adopt a stable ' $\mathrm{C}$ ' posture. If the contralateral shoulder position is used as a command source to control stimulated grasp, a thoracic-lumber-sacral-orthosis (TLSO) may need to be modified to accommodate a sensor mounted to the skin above the sternum. Prophylactic bracing may also influence the ability to cross midline and affect upper extremity performance with or without FNS.

Once the curve progresses past $40^{\circ}$, a spinal fusion is usually recommended. ${ }^{38}$ FNS can be applied after the spinal deformity has been corrected. In fact, five individuals who were found to be appropriate for stimulation in this study had received clinical intervention prior to their screening examinations. These children had undergone surgery to correct spinal curvatures which would have otherwise eliminated them from consideration. All of these individuals successfully participated in the lower extremity program and were able to stand and step with FNS.

Skin involvement prohibited several individuals from participating in the FNS research programs. Prevention of pressure sores is essential for numerous reasons related to the general health and wellbeing of the individual. If pressure sores do occur, conservative measures are preferable before surgical repairs such as muscle flaps. When flaps are indicated, gluteal muscles should be preserved because they are essential for good stimulated standing and walking.

Peripheral denervation was the principal physical impediment to the application of FNS. Without an intact and excitable peripheral nerve, high stimulating currents or long duration stimulating pulses are required to activate the muscle tissue directly. Techniques for activating denervated muscle are generally impractical for functional applications requiring the coordination of many muscles, and may even be dangerous as the chance of an electrical or chemical burn increases. Without the input from reflex arcs and spasms, denervated muscle is also frequently replaced by unexcitable connective tissue. In such cases, the surgical transfer of paralyzed but innervated muscles with excitable LMNs may be possible. Other procedures, such as neurotization, may also be possible to restore the innervation to a denervated muscle.

Peripheral denervation was observed in over one quarter of all the individuals examined. The frequency of LMN injury was similar to that reported for the adult SCI population. In the lower extremity, the highest incidence of denervation was observed in individuals with T12-L3 level injuries. Although this suggests that FNS systems for ambulation or standing may be more applicable to children with lesions above T11, tendon transfers or neurotization are options with much potential that have yet to be explored. The overlapping functions of the upper extremity musculature resulted in preserved innervation of almost $80 \%$ of the finger and thumb extensors and $90 \%$ of the flexors. This suggests that, even though some form of denervation was observed in many children with tetraplegia, a synergist or other muscle with an intact and electrically excitable peripheral nerve may be available as a substitute..$^{27-29}$ When the 10 children with tetraplegia who were considered inappropriate for FNS upon initial evaluation because of LMN damage were reexamined for possible substitution of paralyzed but innervated muscles, four were identified as likely candidates. Although it represents a small sample, and the success of these procedures 
would need to be established, the data suggest that options for FNS exist, even for individuals who exhibit peripheral denervation. According to this limited survey, perhaps as high as $40 \%$ of those with LMN involvement may be able to take advantage of FNS tendon transfer surgery.

For all these reasons, the frequencies of physical or medical complications which interfere with the application of FNS may have been inflated in this study because no preparatory interventions were considered. Conversely, the percentage of children with SCI who may benefit from FNS in the research setting may have been underestimated. In addition, almost $45 \%$ of those children and teenagers examined were still within 1 year post injury. This would suggest that those individuals exhibiting neurological instability at the time of the initial examination may also be appropriate for FNS at some point in the future.

Similarly, complicating psychosocial factors are dynamic and may be addressed with treatment or additional resources. The condition of the individuals initially categorized as inappropriate for FNS may change with time or suitable intervention to the point where they would be better prepared for research participation. Therefore, the findings presented here may be underestimates of the user population of FNS devices. With the proper physical or psychosocial preparation, perhaps more than half of the children with SCI may be made appropriate for FNS. As with the physical data, psychosocial status reflects only the strict inclusion criteria of very specific research programs using experimental devices. It is not meant to imply that clinical applications of FNS should be withheld or denied because of such factors.

The data also seem to indicate that between 40 and $50 \%$ of the children who were classified as completely appropriate for FNS found it acceptable in the research setting. Many postponed participation or were prohibited from taking advantage of the program due to logistical or administrative constraints. Several individuals who initially postponed FNS later decided to participate because admission schedules and transportation issues were resolved. Most individuals in this category were either very young, or unwilling to interrupt their social, home, or school lives to invest the time required to make the commitment to research participation.

Acceptance of the offer of FNS may reflect the research nature of the programs, rather than the functionality or perceived benefits associated with the technology itself. During this study, only percutaneous intramuscular electrodes and devices originally designed to be used with adults were employed. Smaller, lighter and less conspicuous systems that produced more cosmetically pleasing prehension and locomotion may have convinced some candidates to participate. Several physically and psychosocially appropriate candidates expressed the desire to become involved with FNS only after clinical systems were made available. Others were unwilling to undergo implantation of intramuscular electrodes or to maintain the percutaneous interface. These objections may be addressed by the expedient development of fully implantable systems for clinical application similar to pacemakers, provided these patients are less hesitant to have surgery.

The manner in which FNS programs are structured and systems are delivered may also have affected the decision to participate. Because the Shriners program is research-oriented, it may not serve as an accurate model for the acceptability of FNS systems when administered and maintained as a clinical service. The inpatient nature of the lower extremity FNS program was the single most frequently cited reason for rejecting participation. Travel, separation from family, and the need to reside at the hospital are negative aspects of the structure of the research program and should not be associated with the FNS systems themselves. Both upper and lower extremity programs at Shriners draw patients from a wide geographic area, making travel to the hospital for routine examinations or system maintenance extremely inconvenient, even if performed infrequently. Although the upper extremity program is based on an outpatient model, the need to collect data, service the FNS devices, and address other clinical problems associated with system use 
still required volunteers to return to Philadelphia for several days. Reasons for declining or postponing FNS may have been confounded by these programmatic considerations. Investigators and clinicians involved with designing new research or service-oriented programs would be advised to adopt an outpatient model with an emphasis on local participants.

These data do not predict the effectiveness of FNS at achieving the goals of increased function or independence. Nor do they offer any evidence of its success, utility, or ultimate acceptability. They indicate only the relative frequency with which the option of receiving FNS was exercised and do not imply satisfaction with the technology after a period of use. User acceptance of FNS systems will need to be addressed by other means after a large number of systems have been provided for daily use outside of the research setting.

The inclusion criteria for research participation employed in this study may also be unduly restrictive. For example, the criterion regarding tendon transfers was adopted from the CWRU guidelines for adults and may not be appropriate for growing children. The effficacy of active tendon transfer surgery has not been established in the pediatric SCI population, and the effects of growth and maturation on the long term surgical outcome need to be studied before such procedures are recommended preferentially over other clinical options. Parents and families of children with low cervical injuries may view FNS as a desirable alternative if it proves to offer clinical benefits that are similar or superior to surgery. Although the efficacy of FNS in growing children with low tetraplegia also remains to be determined, the feasibility of implementing partial systems in children and teenagers with strong $\mathrm{C} 6$ and $\mathrm{C} 7$ function has been examined. ${ }^{23}$ In a related pilot study at Shriners, working grasps were established in four individuals who did not meet the CWRU criteria because they were candidates for active tendon transfers.

Similarly, FNS may also prove to be beneficial to individuals with high tetraplegia. One teenager classified as physically inappropriate according to the selection criteria for this study because of a $\mathrm{C} 4$ level injury also participated in a pilot program to determine the feasibility of applying FNS to people with high cervical lesions. ${ }^{22}$ Independent control of stimulated grasp and elbow flexion were both achieved in this individual in the laboratory. The success of the pilot study highlights the potential for clinical applications of FNS beyond the limits of the strict inclusion criteria employed in the present study.

Although the sampling process employed in this study was flawed, the results may provide valuable insight into the characteristics of that segment of the pediatric SCI population appropriate for immediate participation in research involving well described neuroprosthetic systems. Since FNS systems for upper and lower extremity function are not yet readily available for clinical use, they are being applied only in highly structured research settings. The first systems to be completely transferred to the clinical environment are likely to be extensions of the most well developed of the experimental devices currently under study. Until research on new clinical applications with other patient populations is complete, and new systems are mature enough for clinical delivery in the future, this sample may offer an initial indication of the characteristics of the pediatric SCI population who may become users of FNS devices in the short term.

Care should be taken in interpreting these results, however, as well as in interpreting the data reported in all published attempts to estimate the FNS user population. Offering preparatory intervention, addressing programmatic and administrative constraints, and relaxing restrictive inclusion criteria for research applications may all serve to increase the estimate of the number of individuals who may eventually benefit from the technology.

\section{Acknowledgements}

The authors would like to thank Maria Frontera, Joy Cohn, Terry Beck, and Eileen Krier for their assistance in collecting the data for this study, and Carolyn Hendrix for her help in 
preparing the manuscript. The contributions of the OT, PT, RT, Nursing and Social Service Departments at the Philadelphia Unit of Shriners Hospitals should also be noted. Special thanks to Drs Peckham and Keith, as well as the entire staff of the Rehabilitation Engineer- ing Center at Case Western Reserve University. We would also like to acknowledge the assistance of Jeanne Teeter of the FES Information Center, Cleveland, Ohio. This work was supported by research grants 15953 and 15954 from Shriners Hospitals for Crippled Children.

\section{References}

1 DeVivo MJ, Fine PR, Maetz HM, Stover SL (1980) Prevalence of spinal cord injury: a re-estimation employing life table techniques. Arch Neurol 37: 707-708.

2 Ergas Z (1985) Spinal cord injury in the United States: a statistical update. Nerv Syst Trauma 2: 19-30.

3 Harvey C, Rothschild R, Asmann A, Stripling T (1990) New estimates of traumatic SCI prevalence: a survey-based approach. Paraplegia 28: 537-544.

4 Stover SL, Fine PR (1987) The epidemiology and economics of spinal cord injury. Paraplegia 25: $225-228$.

5 Stripling TE (1990) Estimates of SCI prevalence. P.S.A. Research Briefs 4: 1-7.

6 Stripling TE (1990) Traumatic spinal cord injury cord demography and etiology. P.S.A. Research Briefs 5: $1-7$.

7 Stover SL, Fine PR (1986) Spinal Cord Injury: The Facts and Figures. The University of Alabama at Birmingham, Birmingham.

8 Bracken MB, Freeman DH, Hellenbrand K (1981) Incidence of acute traumatic hospitalized spinal cord injury in the United States. Am J Epidemiol 113: 615-622.

9 Fine PR, Kuhlemeier KV, DeVivo MJ, Stover SL (1980) Spinal cord injury: an epidemiologic perspective. Paraplegia 17: 237-250.

10 Osenbach RK, Meneze AH (1992) Pediatric spinal cord and vertebral column injury. Neurosurgery 30: 385-390.

11 Cybulski GR, Penn RD, Jaeger RJ (1985) Lower extremity functional neuromuscular stimulation in cases of spinal cord injury. Neurosurgery 15: 132-146.

12 Peckham PH (1987) Functional electrical stimulation: current status and future prospects of applications to the neuromuscular system in spinal cord injury. Paraplegia 25: 279-285.

13 Solomonow M, Baratta RV, Hirokawa S et al (1989) The RGO generation II: Muscle stimulation powered orthosis as a practical walking system for paraplegics. Orthopedics 12: 1309-1315.

14 Andrews B, Baxendale R (1988) A hybrid orthosis incorporating artificial reflexes for spinal cord damaged patients. J Physiol 198: 380 .

15 Kralj A, Bajd T (1989) Functional Electrical Stimulation: Standing and Walking after Spinal Cord Injury. CRC Press, Boca Raton.

16 Kralj A, Bajd T, Turk R, Benko H (1983) Gait restoration in paraplegic patients: a feasibility demonstration using multichannel surface electrode FES. J Rehabil Res Dev 20: 3-20.

17 Yarcony GM, Jaeger RJ, Roth E et al (1990) Functional neuromuscular stimulation for standing after spinal cord injury. Arch Phys Med 70: 201-206.

18 Marsolais EB, Kobetic R (1987) Functional electrical stimulation for walking in paraplegia. J Bone Joint Surg Am 69: 728-733.

19 Smith BT, Mulcahey MJ, Triolo RJ, Betz RR (1991) Pilot study: application of intramuscular stimulation to upper extremity musculature of a child with spastic quadriparesis, cerebral palsy. Proc 13th Ann IEEE EMBS Conf, Orlando, Florida 13: 1814-1816.

20 Hoshimiya N, Naito A, Yajima M, Handa Y (1989) A multichannel FES system for the restoration of more motor functions in high spinal cord injury patients: a respiration-controlled system for multijoint upper extremity. IEEE Trans Biomed Eng 7: 754-760.

21 Nathan RH (1989) An FNS-based system for generating upper limb function in the C4 quadriplegic. Med Biol Eng Comput 11: 549-556.

22 Betz R, Mulcahey MJ, Smith BT et al (1992) Bipolar latissimus dorsi transposition and functional neuromuscular stimulation to restore elbow flexion in an individual with $\mathrm{C} 4$ tetraplegia and $\mathrm{C} 5$ denervation. J Am Paraplegia Soc 15: 220-228.

23 Smith BT, Mulcahey MJ, Triolo RJ, Betz RR (1992) Application of a modified neuroprosthetic hand system in a C7 spinal cord injured child. Paraplegia 30: 598-606.

24 Keith MW, Peckham PH, Thrope GB et al. (1988) Functional neuromuscular stimulation neuroprostheses for the tetraplegic hand. Clin Orthop 233: 25-33.

25 Peckham PH, Keith MW, Freehafer AA (1988) Restoration of functional control by electrical stimulation in the upper extremity of the quadriplegic patient. J Bone Joint Surg Am 70: 144-148.

26 Stroh K, Thrope G, Peckham PH et al (1988) Candidate selection for use with a functional neuromuscular stimulation hand system. Proceedings ICAART, Montreal: 330-331.

27 Freehafer AA, Peckham PH, Keith MW (1988) New concepts on treatment of the upper limb in the tetraplegic: surgical restoration and functional neuromuscular stimulation. Hand Clin 4: 25-33. 
28 Peckham PH, Keith MW, Kilgore KL et al (1992) Surgical alterations and functional electrical stimulation for restoration of hand function. Proc 15th Ann RESNA Conf Toronto: 27-28.

29 Kilgore KL, Peckham PH, Keith MW, Thrope GB (1993) An implanted upper extremity neuroprosthesis. Proc 16th Ann RESNA Conf, Las Vegas, Nevada 404-406.

30 Campbell J, Gerber N, Jursnik J et al (1989) Innervation status of muscles paralyzed by spinal injury. Proc 15th Ann ASIA Scientific Meeting. American Spinal Injury Association, Las Vegas: 97.

31 Campbell J, Meadows P (1992) Therapeutic FES: from rehabilitation to neural prosthetics. Assistive Technol 4.1: 4-18.

32 Peckham PH, Mortimer JT, Marsolais E (1976) Upper and lower motor neuron lesions in the upper extremity muscles of tetraplegics. Paraplegia 14: 115-121.

33 Jaeger RJ, Yarkony GM, Smith RM (1989) Standing the spinal cord injured patient by electrical stimulation: refinement of a protocol for clinical use. IEEE Trans Biomed Eng 36: 720-728.

34 Jaeger RJ, Yarcony GM, Roth EJ, Lovell L (1990) Estimating the user population of a simple electrical stimulation system for standing. Paraplegia 28: 505-511.

35 Heinemann AW (1985) Evaluating the special needs of functional neuromuscular stimulation in research candidates. J Med Eng Technol 9: 167-173.

36 Smilkstein G (1978) The family APGAR. J Family Practice 6: 1231-1239.

37 Betz RR, Beck T, Huss GK et al (1993) Hip instability in children with spinal cord injury. Proc Am Paraplegia Soc Meeting, Las Vegas, Nevada.

38 Dearolf WW, Betz RR, Vogel LC et al (1990) Scoliosis in spinal cord injured patients. J Pediatr Orthop 10: $214-218$ 